EPJ manuscript No.

(will be inserted by the editor)

\title{
Multi-photon corrections to $W$ boson mass determination at hadron colliders
}

\author{
C.M. Carloni Calame ${ }^{1}$, G. Montagna ${ }^{2}$ a, O. Nicrosini ${ }^{1}$, and M. Treccani ${ }^{3}$ \\ 1 Istituto Nazionale di Fisica Nucleare, Sezione di Pavia, and Dipartimento di Fisica Nucleare e Teorica, Università di Pavia, \\ via A. Bassi 6, I-27100, Pavia, Italy \\ 2 Dipartimento di Fisica Nucleare e Teorica, Università di Pavia, and Istituto Nazionale di Fisica Nucleare, Sezione di Pavia, \\ via A. Bassi 6, I-27100, Pavia, Italy \\ 3 Dipartimento di Fisica Nucleare e Teorica, Università di Pavia, via A. Bassi 6, I-27100, Pavia, Italy
}

Received: date / Revised version: date

\begin{abstract}
The impact of higher-order final-state photonic corrections on the precise determination of the $W$-boson mass at the Tevatron and LHC colliders is evaluated. The $W$-mass shift from a fit to the transverse mass distribution is found to be about $10 \mathrm{MeV}$ in the $W \rightarrow \mu \nu$ channel and a few $\mathrm{MeV}$ in the $W \rightarrow e \nu$ channel. The calculation, which is implemented in the Monte Carlo event generator HORACE for data analysis, can contribute to reduce the uncertainty associated to the $W$ mass measurement at present and future hadron collider experiments.
\end{abstract}

PACS. 12.15.Lk Electroweak radiative corrections - 13.40.K Electromagnetic corrections to strong- and weak-interaction processes

\section{Introduction}

In addition to the program of discovery physics, experiments at the high-energy hadron colliders Tevatron RunII and the LHC are expected to pursue the program of precision physics successfully carried out during the last decade at LEP, SLC and the Tevatron itself. In particular, for precision tests of the Standard Model a very precise determination of the $W$ boson mass $M_{W}$ is of particular importance because this would give the possibility, when associated with an improved measurement of the top-quark mass, of putting more severe indirect bounds on the mass of the Higgs boson. The present experimental situation for all the direct measurements of $M_{W}$ is shown in Fig. 1 It is worth noticing that the hadron collider average for $M_{W}$ has an accuracy comparable to the LEP measurement, but even more important is to take into account that the precision expected at the Tevatron is about $30 \mathrm{MeV}$ per experiment per channel at Run IIa and $16 \mathrm{MeV}$ at Run $\mathrm{IIb}$, the latter being the same precision aimed at at the LHC 1,2]. This competes with the precision of the $M_{W}$ measurement expected at a future $e^{+} e^{-}$collider and will correspond to a knowledge of the $W$ mass with a relative precision of $2 \times 10^{-4}$, which obviously requires precise calculations and event generators for the Drell-Yan-like processes $\stackrel{(-)}{p p} \rightarrow W \rightarrow l \nu_{l}$ and $\stackrel{(-)}{p} \rightarrow \gamma, Z \rightarrow l^{+} l^{-}, l=e, \mu$.

\footnotetext{
a Presented by G. Montagna with title "Higher-order QED corrections to $W$ mass determination at hadron colliders"
}

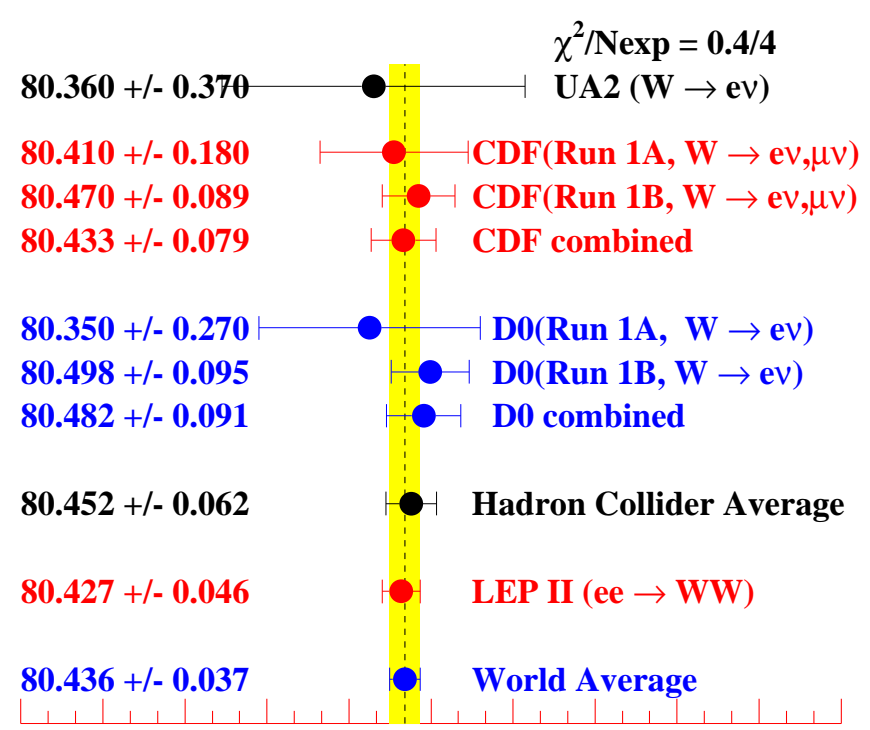

$\begin{array}{lllllllllll}79.5 & 79.7 & 79.9 & 80.1 & 80.3 & 80.5 & 80.7 & 80.9 & 81.1 & 81.3 & 81.5\end{array}$ $\mathrm{Mw}(\mathrm{GeV})$

Fig. 1. Compilation of all the available direct measurements of the mass of the $W$ boson $M_{W}$.

\section{The $W$ mass at hadron colliders}

At hadron colliders the $W$ mass is extracted from the twobody kinematics of the $W$ boson decay into a lepton and a neutrino $W \rightarrow l \nu_{l}$, giving rise to a Jacobian peak in the distribution of the lepton transverse momentum $p_{T}(l)$. 
However, the preferred quantity to determine $M_{W}$ is the transverse mass spectrum $M_{T}$, which is defined as

$$
M_{T}=\sqrt{2 p_{T}(l) p_{T}(\nu)\left(1-\cos \phi^{l \nu}\right)}
$$

where $p_{T}(l)$ and $p_{T}(\nu)$ are the transverse momentum of the lepton and the neutrino, and $\phi^{l \nu}$ is the angle between the lepton and the neutrino in the transverse plane. Actually, the $M_{T}$ spectrum is less sensitive than the $p_{T}(l)$ distribution to the transverse motion of the $W$, which is difficult to model. Having in mind the precision anticipated at the Tevatron and the LHC for $M_{W}$, accurate theoretical predictions including QCD and electroweak radiative corrections are necessary to precisely extract $M_{W}$ from the data. In the presently available calculations [3, 4] $O(\alpha)$ electroweak corrections are included exactly. From these calculations it comes out that electroweak corrections shift the $W$ mass by an amount of the order of $100 \mathrm{MeV}$. In $W$ mass measurements at the Tevatron Run Ib the mass shifts due to radiative effects were estimated to be $-65 \pm 20$ $\mathrm{MeV}$ and $-168 \pm 10 \mathrm{MeV}$ for electron and muon channels, respectively 1,2. From previous studies, it is also known that these shifts are mainly due to final-state photonic corrections because of the presence of large collinear logarithms of the form $\alpha / \pi \log \left(\hat{s} / m_{l}^{2}\right)$, where $\sqrt{\hat{s}}$ is the effective centre of mass (c.m.) energy and $m_{l}$ is the mass of the final-state lepton. Furthermore, the effect of final-state QED corrections significantly depends on lepton identification requirements and detector effects, which are different for electrons and muons. In the presence of realistic selection criteria, the correction due to final-state photon radiation is of several per cent on the $M_{T}$ spectrum in the peak region $M_{T} \approx M_{W}$. This poses the question of the impact of higher-order (i.e. beyond order $\alpha$ ) QED corrections due to the multiple emission of (real and virtual) photons. These higher-order contributions are not presently included in data analysis at the Tevatron but they are estimated to introduce a systematic uncertainty of $20 \mathrm{MeV}$ in the $W \rightarrow e \nu_{e}$ decay channel and $10 \mathrm{MeV}$ in the $W \rightarrow \mu \nu_{\mu}$ decay [1]2. This source of systematic uncertainty is not negligible in view of the foreseen experimental precisions and it can be reduced by means of improved theoretical calculations. Recent work in this direction includes the calculation of the double bremsstrahlung matrix elements $q \bar{q} \bar{q}^{\prime} \rightarrow W \rightarrow l \nu \gamma \gamma$ and $q \bar{q} \rightarrow \gamma, Z \rightarrow l^{+} l^{-} \gamma \gamma$ [5], as well as the calculation of multi-photon corrections to leptonic $W$ decays in the framework of Yennie-Frautschi-Suura exponentiation approach implemented in the Monte Carlo (MC) generator WINHAC [6].

\section{Theoretical approach and numerical results}

In the approach here presented the real plus virtual corrections due to multi-photon radiation are computed in the leading-log approximation using the well-known and wellestablished QED structure-function approach. The corrections are calculated by solving numerically the DGLAP equation for the electron structure function by means of the QED Parton Shower algorithm developed in Ref. [7. Only radiation from the final-state leptons is presently included in our approach (by simply attaching a single QED structure function to the final-state lepton), because it is known that quark-mass singularities, originating from initial-state photon radiation, can be reabsorbed into a redefinition of the Parton Distribution Functions (PDFs), in analogy to gluon emission in QCD 8. After this massfactorization procedure, initial-state-radiation has only a small and uniform impact on the $M_{T}$ spectrum, while final-state radiation significantly distorts the shape of the $M_{T}$ distribution, affecting in turn the $M_{W}$ extraction. The formulation is implemented into a MC generator for data analysis, HORACE (Higher Order RAdiative CorrEctions) 9. It is interfaced to PDFs and incorporates lepton identification criteria and detector resolution effects, in order to perform simulations for the hadronic process $\stackrel{(-)}{p p} \rightarrow W \rightarrow l \nu, l=e, \mu$ as realistic as possible. HORACE can calculate photonic corrections to all orders and at order $\alpha$, to disentangle the effect of higher-order contributions and to compare with the available $O(\alpha)$ programs. A first round of comparisons was performed with WGRAD [3] in Ref. 9], showing good agreement. A more detailed analysis, based on the comparison between HORACE and WINHAC, is in progress [10.

To evaluate the shift on the fitted $W$ mass induced by higher-order QED final-state corrections we used HORACE and performed $\chi^{2}$ fits to MC pseudo-data for the $M_{T}$ spectrum, simulating acceptance cuts, lepton identification criteria and detector resolution effects as reported in Ref. [9]. The c.m. energy considered in our study is $\sqrt{s}=2 \mathrm{TeV}$, corresponding to the Tevatron Run II data taking, but we checked that the conclusions of our analysis do not change at the LHC energy of $\sqrt{s}=14$ $\mathrm{TeV}$, when using the same cuts and detector specifications. The basic steps of the fitting procedure are the following: i) we generate a sample of pseudo-data including $O(\alpha)$ corrections for a reference value of the $W$ mass, $M_{W}^{r e f}$, and we simulate the $M_{T}$ spectrum within the fit region $65 \mathrm{GeV}<M_{T}<100 \mathrm{GeV}$; ii) we consider $N$ different $W$ mass values around $M_{W}^{r e f}$ and we generate $N m_{T}$ spectra including higher-order corrections; iii) for each $M_{W}$ value, we calculate the $\chi^{2}$ as

$$
\chi^{2}=\sum_{i}\left(\sigma_{i, \exp }-\sigma_{i, \alpha}\right)^{2} /\left(\Delta \sigma_{i, \exp }^{2}+\Delta \sigma_{i, \alpha}^{2}\right)
$$

where $\sigma_{i, \alpha}$ and $\sigma_{i, \exp }$ are the MC predictions for the $i^{t h}$ bin at the $\mathcal{O}(\alpha)$ and exponentiated level, respectively, and $\Delta \sigma_{i, \alpha}, \Delta \sigma_{i, \exp }$ the corresponding statistical errors due to numerical integration. We derive in conclusion the $M_{W}$ shift due to higher-order effects looking at the minimum of the $\chi^{2}$ distribution. The results of our analysis are reported in Fig. 2] showing the $\Delta \chi^{2}=\chi^{2}-\chi_{\text {min }}^{2}$ distributions as a function of $\Delta M_{W} \equiv M_{W}-M_{W}^{r e f}$. It can be seen that the mass shift due to higher-order effects is about $10 \mathrm{MeV}$ for the $W \rightarrow \mu \nu$ channel (solid line) and a few $\mathrm{MeV}$ (dashed line) for the $W \rightarrow e \nu$ channel, as a consequence of the different identification requirements for 


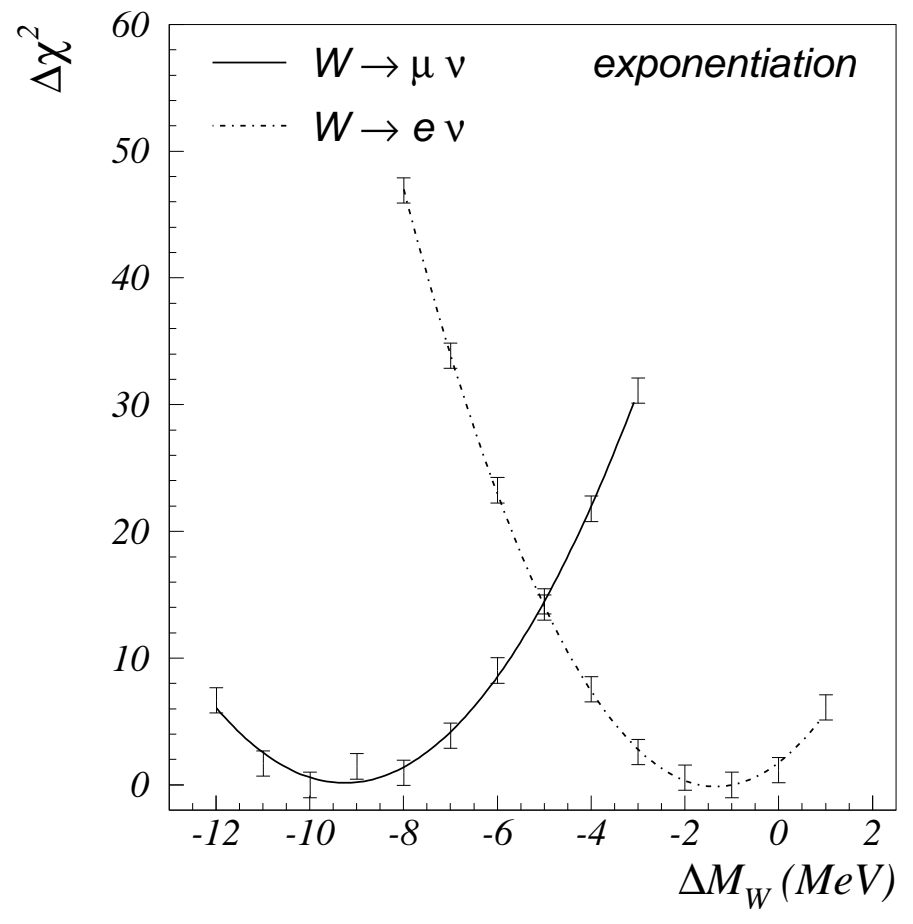

Fig. 2. The $\Delta \chi^{2}=\chi^{2}-\chi_{\min }^{2}$ distributions from a fit to the $M_{T}$ spectrum as a function of the $W$ mass shift $\Delta M_{W}$ due to higher-order QED final-state corrections, at $\sqrt{s}=2 \mathrm{TeV}$. The results for the $W \rightarrow e \nu$ and $W \rightarrow \mu \nu$ channels are shown.

electrons and muons. Therefore, in view of the expected precision of $15-30 \mathrm{MeV}$ for $M_{W}$ at the Tevatron Run II and at the LHC, it will be important to take multi-photon effects into account when extracting $M_{W}$ from the data.

\section{Conclusions and outlook}

In view of improved precision measurements of the $W$ mass at hadron colliders, we calculated higher-order QED final-state corrections to the process of single $W$ production in hadronic collisions. We found that the shift due to these corrections is about $10 \mathrm{MeV}$ in the $W \rightarrow \mu \nu$ channel and a few $\mathrm{MeV}$ in the $W \rightarrow e \nu$ channel. The calculation, if included in future experimental analyses, would reduce the uncertainty in the precision measurement of the $W$ mass at hadron colliders. To this end, the Monte Carlo program HORACE is available for data analysis. We noticed that the shifts significantly depend on particle identification requirements and detector effects. Therefore, a more precise assessment of our conclusions would require a full detector simulation, which needs for collaboration with experimental colleagues. Since $Z$ boson parameters, such as the $Z$ boson mass and width, are crucial in determining the lepton energy and momentum scales, which enter and affect the $M_{W}$ measurement, a calculation of higher-order corrections to the neutral-current process $\stackrel{(-)}{p} \rightarrow \gamma, Z \rightarrow l^{+} l^{-}$ is needed, being the $O(\alpha)$ electroweak corrections to the above process already known in the literature [11. Work is in progress in this direction.

\section{References}

1. R. Brock et al., hep-ex/0011009

2. U. Baur, hep-ph/0304266

3. U. Baur, S. Keller and D. Wackeroth, Phys. Rev. D59 (1999) 013002.

4. S. Dittmaier and M. Krämer, Phys. Rev. D65 (2002) 073007.

5. U. Baur and T. Stelzer, Phys. Rev. D61 (2000) 073007.

6. W. Placzek and S. Jadach, hep-ph/0302065

7. C.M. Carloni Calame et al., Nucl. Phys. B584 (2000) 459;

C.M. Carloni Calame, Phys. Lett. B520 (2001) 16.

8. H. Spiesberger, Phys. Rev. D52 (1995) 493.

9. C.M. Carloni Calame, G. Montagna, O. Nicrosini and M. Treccani, hep-ph/0303102

10. C.M. Carloni Calame, G. Montagna, O. Nicrosini, W. Placzek and S. Jadach, in preparation.

11. U. Baur et al., Phys. Rev. D65 (2002) 033007. 